\title{
Special Issue: Dynamic Data-Driven Applications Systems (DDDAS) Concepts in Signal Processing
}

\author{
Erik Blasch $^{1} \cdot$ Shashi Phoha ${ }^{2}$
}

Published online: 24 May 2017

(C) Springer Science+Business Media New York (outside the USA) 2017

Dynamic Data Driven Applications Systems (DDDAS) is a transformative framework for incorporating evolving data into a dynamic system to adapt to operational conditions, and recursively to steer its measurement components that generate such data. Signal processing techniques are applied to big data analysis to spawn developments in designs, algorithms, architectures, and applications. DDDAS is based on four interactive concepts: applications modeling, mathematical and statistical processing, measurement systems, and systems software design, as shown in Fig. 1. Rooted in control theory, many of the prominent developments include methods of signal processing to determine the relevant data for model updates. These model updates arise from statistical data analysis. New insights have been developed for measurement systems with unobservable data, such as new architectures to emulate data collections for missing data. To complement the modeling and statistical analysis, software methods are paramount for realtime applications. With recent advances from Very Large Scale Integration (VLSI) and high performance computing (HPC), implementations of DDDAS concepts are realizable. The DDDAS framework has spawned numerous applications such as environment analysis (e.g. weather); robotic systems (e.g., unmanned aerial vehicle, unmanned ground vehicle (UAV/ UGV) coordination); image processing (e.g., target tracking),

Erik Blasch

erik.blasch@gmail.com

Shashi Phoha

Sxp26@psu.edu

1 Air Force Research Laboratory, 525 Brooks Rd, Rome, NY 13441, USA

2 Applied Research Laboratory, Pennsylvania State University, P.O. Box 30, State College, PA 16804, USA and embedded computing (e.g., hardware/software designs). This special issue brings together DDDAS examples for the readers. More information can be found at www.1dddas.org.

The special issue received numerous submissions and two were selected based on the implementation of the entire DDDAS framework presented in Fig. 1.

The first was S. Sarkar, et al., "Deep Learning for Automated Occlusion Edge Detection in RGB-D Frames" (10.1007/s11265-016-1209-3). The authors addressed the challenge of real-time video tracking when objects are occluded. Using Deep Convolutional Neural Networks (CNN) algorithms from computer vision, they demonstrate robust occlusion edge determination via DDDAS processing of heterogeneous measurements from visual cameras, depth modeling, and motion models. The system level performance application was executed in experiments using parallel computing software with Graphical Processor Units (GPUs) from the Compute Unified Device Architecture (CUDA) reducing the false alarms from occlusion detection.

The second was R. Wu, et al., "A Container-based Elastic Cloud Architecture for Pseudo Real-time Exploitation of Wide Area Motion Imagery (WAMI) Stream" (10.1007/ s11265-016-1206-6)". The authors addressed the difficulty of using large scale data as demonstrated with WAMI. The paper highlights software implementation solutions by using a cloud-based approach to decompose the imagery and enhance multi-target tracking. Their DDDAS approach included the Pseudo Real-time Exploitation of Sub-Area (PRESA) framework which divides the data into spatial regions for parallel processing of video tracking algorithms. Key to the approach was the stitching of tracks from the spatial regions. The results demonstrate that the virtual-machine container approached improved the processing frame rate, object detection, and tracking accuracy, as compared to approaches using the Hadoop method. 


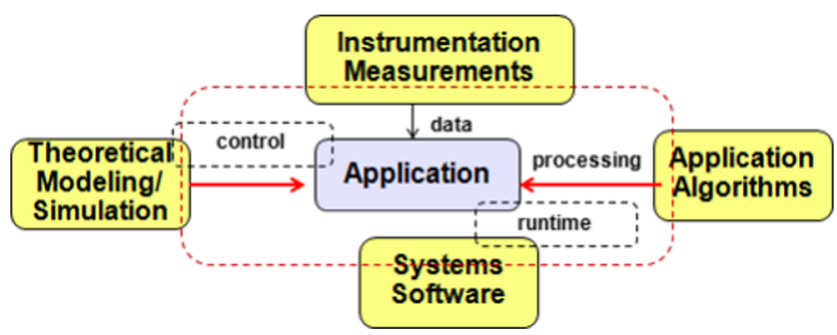

Figure 1 Dynamic data-driven applications systems (DDDAS) framework.

The guest editors believe these papers demonstrate solutions using the DDDAS framework to advance the field of signal processing systems and hope they stimulate further development of DDDAS applications. The editors express their appreciation to the authors and reviewers for contributing to this special issue.

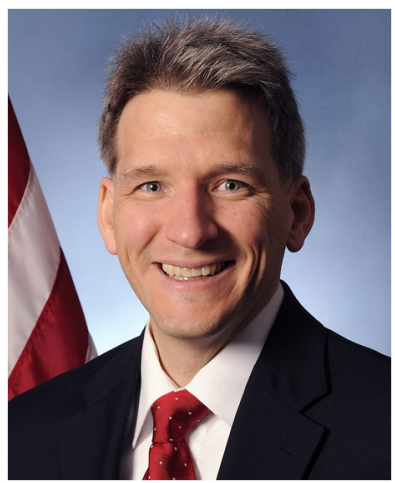

Erik Blasch is a principal scientist at the the United States Air Force Research Laboratory (AFRL) in the Information Directorate at Rome, NY, USA. From 2009 to 2012, he was an exchange scientist to Defence Research and Development Canada (DRDC) at Valcartier, Quebec. From 2000 to 2009, Dr. Blasch was the Information Fusion Evaluation Tech Lead for the AFRL Sensors Directorate COMprehensive Performance Assessment of Sensor Exploitation (COMPASE) Center supporting design evaluations in Dayton, OH. Dr. Blasch has been an Adjunct Electrical Engineering Professor at Wright State University teaching signal processing, target tracking, and information fusion. Dr. Blasch served on the IEEE Aerospace and Electronics Systems Society (AESS) Board of Governors (2011-2016), was a founding member of the International Society of Information Fusion (ISIF) (www.isif.org), and the 2007 ISIF President. He has focused on information fusion, target tracking, pattern recognition, and robitcs research compiling $650+$ scientific papers and book chapters. He holds 14 patents, presented over 30 tutorials, and is an associate editor of three academic journals. His books include High-Level Information Fusion Management and Systems Design (Artech House, 2012) and Context-Enhanced Information Fusion (Spriner, 2016), and Multispectral Image Fusion and Colorization (SPIE, 2017). Dr. Blasch received his B.S. in Mechanical Engineering from the Massachusetts Institute of Technology in 1992 and Master's Degrees in Mechanical ('94), Health Science ('95), and Industrial Engineering (Human Factors) ('95) from Georgia Tech and attended the University of Wisconsin for a $\mathrm{MD} / \mathrm{PhD}$ in Neurosciences/Mech. Eng until being called to military service in 1996 to the United States Air Force. He completed an MBA ('98), MSEE ('98), MS Econ('99), and a PhD ('99) in Electrical Engineering from Wright State University and is a graduate of Air War College ('08). He is the recipeint of the IEEE Bio-Engineering Award (Russ-2008), IEEE AESS Magazine Best Paper Award (Mimno-2012), Military Sensing Symposium Ledaership in Data Fusion Award (Mignogna-2014). He is a Fellow of SPIE, Associate Fellow of AIAA, and a senior member of IEEE.

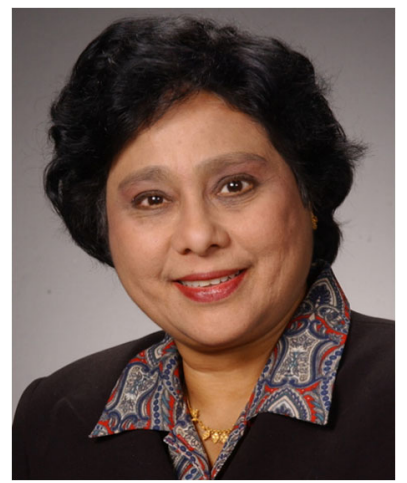

Dr. Shashi Phoha is the Director of the Information Science \& Technology Center of ARL and Graduate Faculty of Electrical Engineering at Penn State, since 1991. She has also been the Director of The Information Technology Laboratory of NIST, Director of Information Analysis Division of Computer Sciences Corporation, and Department Head at ITT. Her research focuses on multi-sensor data fusion, machine learning and autonomy, combining data science, operations research, automata theory, dynamic systems, and control theory for networked intelligence. She was awarded the 2004 Technical Achievement Award by the IEEE Computer Society for her contributions to intelligent networked systems automation. She has published over 200 research papers, three books, several book chapters, and has several patents licensed to industry. She was associate editor of IEEE SMC and is currently Chief Editor of Frontiers in Robotics: Sensor Fusion and Machine Perception, and Editor of the International Journal of Distributed Sensor Networks. 